\title{
6G Mobile Communications for Multi-Robot Smart Factory
}

\author{
Zhenyi Chen ${ }^{1}$, Kwang-Cheng Chen ${ }^{1, *}$, Chen Dong ${ }^{2}$ \\ and Zixiang $\mathrm{Nie}^{1}$ \\ ${ }^{1}$ University of South Florida, Tampa, FL, USA \\ ${ }^{2}$ Fuzhou University, Fuzhou, Fujian, China \\ E-mail: zhenyichen@usf.edu; kwangcheng@usf.edu; dongchen@fzu.edu.cn; \\ znie@usf.edu \\ ${ }^{*}$ Corresponding Author
}

Received 16 May 2021; Accepted 03 October 2021;

Publication 16 December 2021

\begin{abstract}
Private or special-purpose wireless networks present a new technological trend for future mobile communications, while one attractive application scenario is the wireless communication in a smart factory. In addition to wireless technologies, this paper pays special attention to treat a smart factory as the integration of collaborative multi-robot systems for production robots and transportation robots. Multiple aspects of collaborative multi-robot systems enabled by wireless networking have been investigated, dynamic multirobot task assignment for collaborative production robots and subsequent transportation robots, social learning to enhance precision and robustness of collaborative production robots, and more efficient operation of collaborative transportation robots. Consequently, the technical requirements of $6 \mathrm{G}$ mobile communication can be logically highlighted.
\end{abstract}

Keywords: Smart factory, industry 4.0, smart manufacturing, wireless communication, uRLLC, mMTC, 5G, 6G, multi-robot system, multi-agent system, collaboration, artificial intelligence, machine learning, reinforcement learning, social learning, security.

Journal of ICT Standardization, Vol. 9_3, 371-404.

doi: 10.13052/jicts2245-800X.934

(C) 2021 River Publishers 


\section{Introduction}

With massive machine-type communication (mMTC) and ultra reliable and low-latency communication (uRLLC) as the two pillar technologies [1-3], private networking has emerged as a penitential application scenario for $5 \mathrm{G}$ and beyond. In light of the nature of mMTC and uRLLC, and rapid advances of artificial intelligence (AI), communication for collaborative smart machines such as autonomous vehicles and robots suggests a new communication scenario, communication for AI, which applies private or special-purpose mobile network architecture.

As mobile communication and AI shall elevate human society to digital society, Industry 4.0, particularly smart manufacturing, indicates further satisfaction of human life along this trend.

Smart manufacturing aims to facilitate the production flow and process more flexible, versatile, and adaptable to the rapid-changing market demands $[4,5]$. Smart manufacturing plays an important role in Industry 4.0, covering three aspects, supply-demand, logistics and smart factory [4]. The major technological challenge lies in realization of smart factories. As [6], robots play the central role in industrial engineering. Furthermore, collaborative robots are necessary to implement the smart industrial systems, to form a complex robotic ecosystem of dynamic motions in AI control [7]. Considering the fundamental functionalities and in-feasibility of centralized control, a smart factory can be viewed as collaborative multi-robot systems of two classes of collaborative robots, production robots and transportation robots [4].

As illustrated in Figure 1, customers' demands and market trends are analyzed in the cloud side. Market analysis is generated with big data. With the logistics information from the supply chain production plan can be developed and adjusted. For smart manufacturing, the production plan can be dynamically adjusted at any time [8]. With the advance of wireless communication, the smart factory is flexible to be reconfigured and adjusted to form a production flow according to the users requirements. Moreover, wireless communication is more robust to the harsh external environment in the factory, such as strong electromagnetic, critical heat, undamped vibrations and humidity $[9,10]$. Better cost efficiency also promote the wide use of wireless communication in smart factory.

In the past few years, the fifth generation (5G) mobile communication technology has made revolutionary progress and become the main technology of wireless communication [11]. The $5 \mathrm{G}$ is expected to network all 


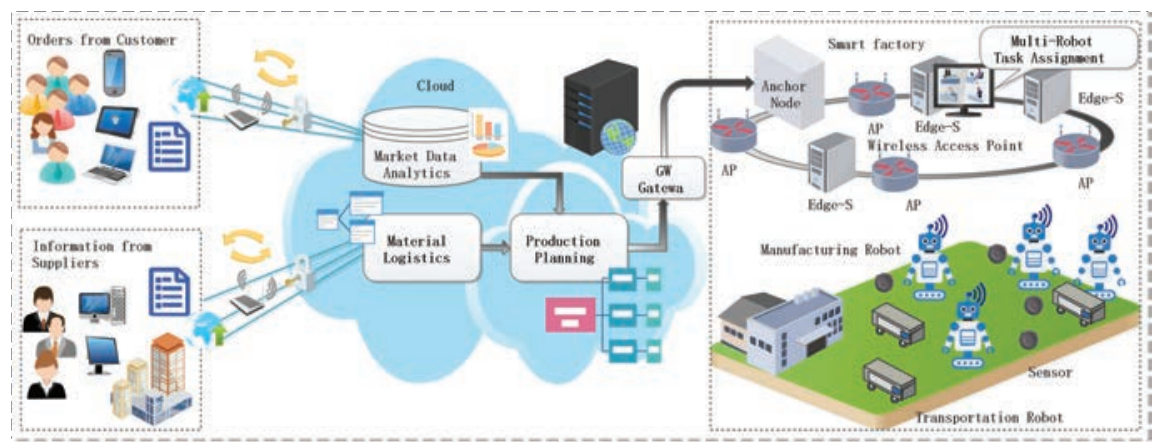

Figure 1 Demand-driven mass customized smart manufacturing in Industry 4.0.

kinds of devices, sensors, actuators and machines in the factory [11-13]. However, smart factory put forward significant challenges to the wireless communication especially to the reliability and latency. The requirement for real-time configuration to production robots, sensor fusion for mobile robot, and collaborating among multi-robot systems, which require a higher datarate, more reliability, and ultra low-latency may exceed even the capabilities of the $5 \mathrm{G}$ systems. The networked robots serve as critical sources of big data that helps inform decision making in the multi-robot systems [4]. The reliable high-throughput connectivity across thousands of devices with submillisecond response times in the smart factory. How to innovate wireless technology to meet the evolving and stringent requirements of smart factory presents a technology opportunity for the sixth generation $(6 \mathrm{G})$ mobile communication $[4,13,14]$.

The main goal of this paper is we present a frame of smart factory as multi-robot systems that incorporates production robots and mobile transportation robots, wireless networks, sensors, and clouds. The smart factory can be view as wireless networked multi-robot systems to realize customization with the support of collective intelligence and mobile intelligence. Due to the limit of $5 \mathrm{G}$ wireless communication, $6 \mathrm{G}$ wireless communication is regarded as key feature to realizing smart factory. The paper analyzes the requirements of the wireless network technology and concludes the smart factory as a use case of $6 \mathrm{G}$ wireless communication.

The organization is as follows: Section 2 puts forward 5G and 6G technologies for smart factories. A scenario of smart factory as wireless networked multi-robot systems is illustrated in Section 3. The flexible production flow is enabled by wireless network technology is presented in 
Section 4. Requirements to $6 \mathrm{G}$ to improve collaborative multi-robot systems are discussed in Section 5 and 6 respectively. Section 7 is the conclusion.

\section{$25 \mathrm{G}$ and Beyond 5G Technology for Smart Factories}

The 5G mobile communication techniques were developed target at the critical industrial applications such like factory automation. The three key components are enhanced mobile broadband (eMBB), massive machine-type communication (mMTC), and ultra-reliable and low-latency communication (uRLLC). The factory automation has been greatly benefited from the radical changes by the most recent $5 \mathrm{G}$ development $[4,10,13]$. Several applications of industrial wireless communication have been studied [10, 15-17]. AIbased technologies are the typical feature of $6 \mathrm{G}$ network $[11,18]$ which aims to meet rigorous ultra reliable and low latency communications wireless network service needs, and beyond, achieving high flexibility, reliability, security and energy efficiency [19]. With the key features, such like high bandwidth, mobility, reliability, and low latency, the application prospects of $5 \mathrm{G}$ and beyond wireless communication technology in smart factory is clearly a trend.

\subsection{Massive Machine-type Communication}

Machine-Type Communication (MTC) have been developed in 3GPP (3rd Generation Partnership Project) [1,20]. The technical challenge is deal with massive number of connections from MTC devices and the extension of coverage of MTC devices in a large geographical environment [2]. To satisfy the needs of factory automation, each device may play multiple roles among the sensors, the decision maker and the action executor. Therefore, the ultimate goal of Machine to Machine (M2M) communications is to provide scrupulous connections among all MTC devices [20]. A key feature of the Internet of Things (IoT) is the huge number of connected devices which is the first challenge to deal with [21]. Several papers have studied the mMTC (massive Machine Type Communications) application for the smart city and smart manufacturing which relied on sensing and monitoring with applying the IoT [22-27].

Smart factory is an important application of mMTC [28] developed a simple mode to understand how MTC can be applied for the smart factories. The paper indicated a base station is able to support up to 1400 MTC devices with no more than $70 \mathrm{~ms}$ access delay and the 0.9 success rate by 
using RACH channel and $1 K$ bits per packet.Study shows there are still not sufficient to satisfy the requirements for reliable information and data privacy in most scenarios of smart factories. The researchers are looking into a new generation of wireless networks, such like $6 \mathrm{G}$ communication, to meet the need for fully connected, intelligent factory $[13,29]$.

\subsection{Ultra-reliable and Low-latency Communication}

As one of the important technologies in 5G, Ultra-reliable and Low-latency Communication (uRLLC) supports the mission-critical applications, such as factory automation and autonomous vehicles. Low-latency communication allowing faster uplink and downlink transmissions by introducing short transmission time slots. This reliable transmission is for the requirement of closed-loop control of robots in the smart factory. The safety and reliable deployment of autonomous vehicles also must rely on ultra-low latency wireless networking to keep end-to-end networking latency down to $1 \mathrm{~ms}$ range [3]. Real-time communication services for delivering control, sensing and actuation information are typical application scenarios of uRLLC so that end-to-end networking latency becomes more and more important than other features in Quality-of-Service (QoS) in wireless networking [22-27,30,31].

In the smart factory, the requirements for real-time control are important for both the production robots and transportation robots. smart factory for Industry automation examples with time-critical process optimization are discussed in [32-34]. In such application scenarios, the information of the sensors about their status need to be delivered to the controller and actuator within a latency less than $1 \mathrm{~ms}$ for the real-time control. Several papers also discussed the issues of the network control under uncertainty [35-37]. The requirements of the communication, such as delay and packet loss, can be quite extreme and must be taken into consideration when deploy wireless network technologies $[38,39]$.

\section{Smart Factory as Wireless Networked Multi-robot Systems}

The development of information technology has promoted the development of Industry 3.0, especially the automated production line has adopted robots instead of human labor for mass production. In factory automation, tasks are assigned to production robots following certain sequences for different products. The production robots form a multi-robot system (MRS) that collaborate 
for same production goal. Another team of robots in is transportation robots, which is also known as autonomous guided vehicles (AGVs), that also form an important part of the MRS [4].

In Industry 4.0, the smart manufacturing tends to provide smaller batch production according to the demand of different users [40-42]. A wider range of technology namely artificial intelligence (AI) robotics, Industrial Internet of Things (IIoT), intelligent sensors, augmented reality (AR), cloud computing, edge computing, big data, and digital fabrication, which will facilitate smart factory that aim at the rapid production of variety of small batches products. In such a smart factory, the production lines will be in a more flexible way that are required to work in a more complex, less structured and subject to more frequent changes than the fixed production lines in Industry 3.0. The robots in the smart factory should be able to collaborate with each other thus form a wireless networked multi-agent system (MAS) [43, 44].

Depend on demands from remote customers, smart factory defines the production goals and executes high flexible and efficient multi-robot operations $[4,45]$. These operations refer to multi-robot tasks assignment (MRTA), energy-efficiency production, robots collaboration based on computing, etc. $[5,46]$, mainly involved in production robots and transportation robots [4]. Each robot (agent) makes decisions locally and collaboratively with others through wireless networks [47] to realize the flexible and efficient mass customized production. Essentially, smart factories are constructed by wireless networked multi-robot systems.

\subsection{Holistic View of Smart Factory in Industry 4.0}

A key feature of smart factory is the flexible and adaptable of the production. Changing demands from customers require rapid response from the smart manufacturing in Industry 4.0. Flexible arrangement of production flows for several products will be made to facilitate smart manufacturing. The smart manufacturing process consists of three main stages as Figure 1 described. Firstly, a portfolio of products is generated based on the online big data analysis on the market. Secondly, the logistics of the acquired components and materials are executed through the online methodology. Finally, the production goal and arrangement of multi-robot processes are developed for the production in the smart factory $[5,45]$. The production is dynamically adjusted to different user demands from the market in a real-time way with the support by big data, artificial intelligence, robotics, and wireless networking technology. 


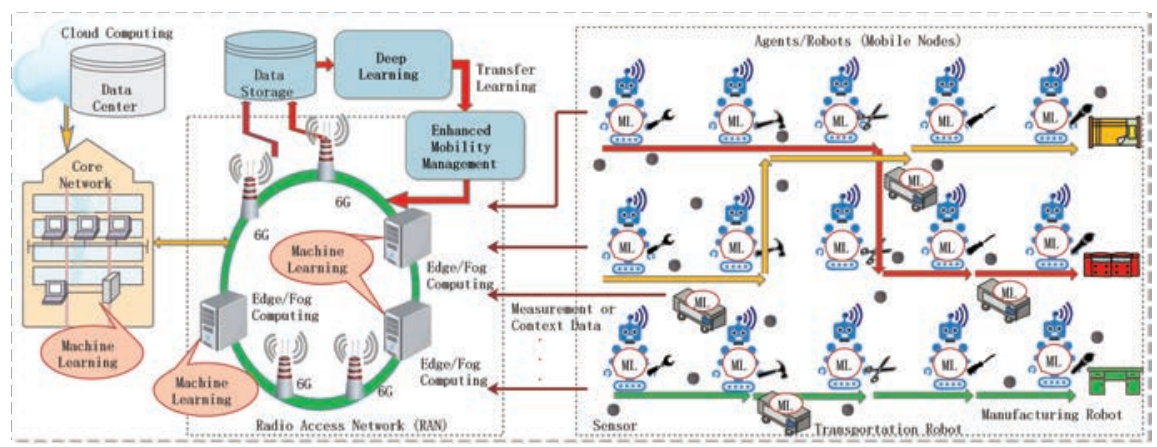

Figure 2 The holistic architecture of a smart factory enabling 6G mobile communications.

Undoubtedly, smart factory is the key component of smart manufacturing. Figure 2 gives the holistic architecture of a smart factory enabling 6G mobile communications. The smart factory consists of four parts, core network, radio access network (RAN), offline machine learning network, and mobile nodes (agents/robots).

There are two classes of robots that enable the flexible production in smart factory. One class is the production robots on production lines that provide the adaptability of production. The other is the AGVs that enable flexible production flows in the smart factory. These robots in smart factory form multi-robot systems (MRSs). In other words, the production robots and transportation robots work together for the maximum flexibility and high efficiency. These robots receiving instructions from the control center, making decisions based on data, or collaborating with other robots are all through the wireless network.

What's more, the communication between the entire smart factory and the decision data from customer demands, the communication between factory and the remote-end cloud computing devices or the near-end edge/fog computing devices, and the communication between factory and the logistics all need to rely on wireless networks.

\subsection{Reconfigurable Production Lines}

The cooperative assembly methodologies are leading the industry automation. A production line usually consists of the cooperate assembly robots. The traditional control systems are not capable of high-level adaptability. The robots on the production line is set up in a fixed way for a certain product for mass production and lack intelligent design to fulfill the requirement of 


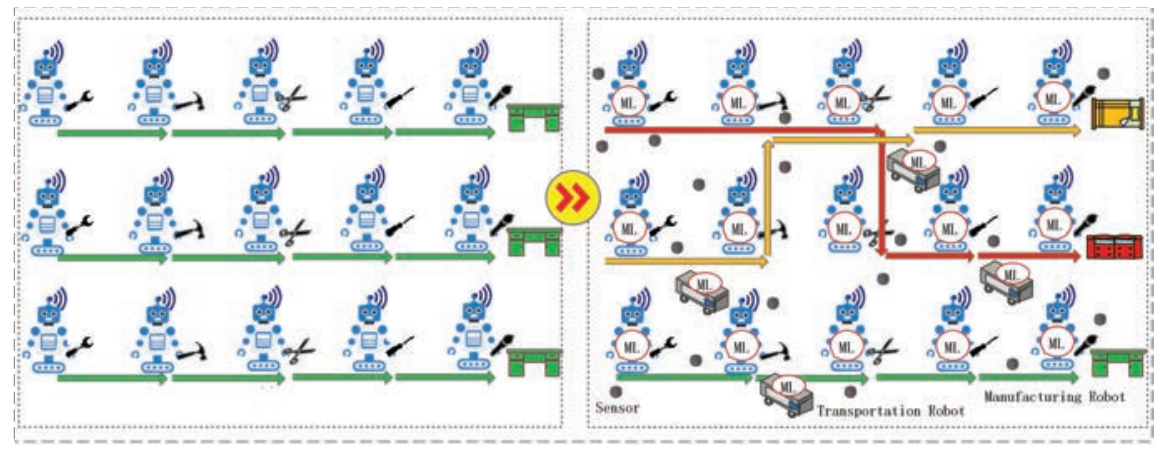

Figure 3 Reconfigurable production lines.

dynamic reconfiguration [41,42]. For smart manufacturing, it is necessary to reduce configure time and cost for robots that are engaged in more frequent changes than the traditional fixed production line [40]. Sensors and software capabilities are making the new generation of robots smarter [40,41]. The manufacturing robots are becoming easier for automatic reconfiguring and reprogramming so as to deal with the frequent assignment of assembly tasks. The networked control and deep learning and reinforcement learning skills will be applied to enable the production lines to adapt to the uncertainties and variations in the real environment.

Figure 3 illustrates the reconfigurable production lines in a smart factory. The left sub-figure is the fixed configuration production lines. The three production lines produce the same products with the same appearance, same color, and same characteristics. To achieve flexible production and maximum utility, the sub-figure on the right is the reconfigurable production lines. With the time-varying production strategy, these robots collaborate to complete different products [4] relying on available resources. Consequently, the demand-driven mass customized production can be meet by the reconfigurable production lines.

There are end-effectors which are the working end of the robot arms to execute specific functions. There can be more than fifty cables in motion on the production robot that could be break during the repeated motion of the robot arms. The unexpected breakages of cables will cause serious cost to maintain of production line plus the loss of production. Wireless networks thus are the preferred solution for the production robots in the smart factory. Wifi operating in the unlicensed frequency band could be the preferred solution to the communication method for the control of the robots because of the lower cost $[9,48]$. 


\subsection{Autonomous Guided Vehicles}

During the production in the factory, cost to transport materials, components, and semi-products is always one of the major consideration due to the distributed nature of manufacturing [49-51]. Autonomous guided vehicles (AGVs) are trackless and generally navigated on the floor of the workshop. Due to the absence of track, the AGVs system can be realized completely with wireless network technologies [29,52].

The manufacturing response time is also very important to the efficiency of production. In the smart factory, AGVs are widely adopted in the transportation robot system to provide the capability of transport by real-time monitoring, connectivity restore, and collaborative control. The advances of low-latency communication technologies allow AGVs to communicate in a very efficient way [48] presented real-time AGVs logistic sorting system in a factory automation application that use. With the routes and data transmission of AGVs optimized centrally by a control center, the moving AGVs report their locations to the center and receive the scheduling paths [9] presented an example of wireless AGVs in a smart factory. IEEE 802.11 (WIFI) in the unlicensed 2.4- $\mathrm{GHz}$ frequency band is used for the communicatin between AGVs and the control center [53] discussed an application of the AVGs that use radio frequency identification for interactions. AGVs thus can be used for the introduction of reconfiguration capabilities and thus form the flexible production flow in smart factory which will improve the performance effectively [54].

Figure 3 shows the work scene of transportation robots. Their tasks are delivering the semi-products to manufacturing robots to form the flexible production flow. The scenarios of AGVs in smart manufacturing show that energy efficiency of control on AGVs should be considered.

\subsection{Wireless Network in a Smart Factory}

To accomplish the flexible production in smart factory, the reconfigurable production lines, AGVs, and sensors can be organized in ad hoc teams for a given production goal [17] discussed a time-sensitive industrial networking protocol stack for communications [55] proposed a reliable an fault-tolerant embedded control system for factory automation using Ethernet as the network technology [31] indicted that the high-performance communication place a key role in time-sensitive industrial networks. Such technologies leverage the application of both production robots and AGVs which are deployed in a large number in smart factory. Many Industrial Internet of 
Table 1 Key performance requirements to the wireless communications in smart factory

\begin{tabular}{lcccc}
\hline Technical & IMT-2020 & Motion Control & Mobile Robots & Manufacturing \\
Requirements & {$[60]$} & {$[61]$} & {$[61]$} & {$[57]$} \\
\hline Latency & $1-10 \mathrm{~ms}$ & $<1 \mathrm{~ms}$ & $<1 \mathrm{~ms}$ & 0.1 ms minimum \\
Data rate & $10-100 \mathrm{MB} / \mathrm{s}$ & Low & $>10 \mathrm{Mbps}$ & $\mathrm{NA}$ \\
Reliability & $\mathrm{NA}$ & $\mathrm{NA}$ & $\mathrm{NA}$ & $10^{-9}$ \\
Mobility & $350-500 \mathrm{~km} / \mathrm{h}$ & Low & $<20 \mathrm{~m} / \mathrm{s}$ & Low \\
Density & $10^{4}-10^{6}$ devices $/ \mathrm{km}^{2}$ & High & Low & $20-200$ Nodes per cell \\
Energy efficiency & $\geq 90 \%$ & $\mathrm{NA}$ & High & High \\
\hline
\end{tabular}

Things (IIoT) architectures and standards have been proposed based on the communication and networking technologies $[53,56]$. Without ultra-reliable and low latency wireless networking, flexible MRTA in a smart factory is not possible $[11,14,57]$. The main technical requirements are low latency, reliability, mobility, data rate, density (number of nodes/sensors), energy efficiency were suggested as bellow [57-62].

The available wireless communication technologies could be coordinated to be solutions for the challenges. Among them, WIFI, Bluetooth, and ZigBee are the most popular communication technologies for the AGVs and production robots in the smart factory. While the low-range communication, like NFC and RFID, could be good ways for short distances data exchange. Table 1 shows some key performances requirements to wireless networking in smart factory suggested by the researchers from academia and industry. $5 \mathrm{G}$ new radio wireless cellular system can not fulfill the requirements of high-precision real time communication. Additional communication technologies are needed for realizing smart factories [63]. The transmission delays that cause the end-to-end (E2E) delay still be the bottlenecks for wireless communication in smart factory. Researchers believe the so-called $6 \mathrm{G}$ communication should guarantee the challenges in wireless network design that make smart factory as a use case for $6 \mathrm{G}[11,13,64]$.

\section{Real-time MRTA}

Multi-robot task assignment (MRTA) problem in smart factory is assigning the manufacturing operations to the collaborate robots on a production line. The production processes in a factory typically follows a certain flow. The solutions to MRTA in factory automation are restricted to the spacial constrain due to the fixed production lines which means a traditional MRTA considers optimal assignment among the production robots. The smart factory is enabled by wireless networking with adaptability for dynamical appearing production plans. By dynamically deploy the production robots 
and AGVs, flexible production in smart factory can be model as a real-time MRTA problem that will consider the costs to deploying both production robots and AGVs [4].

\subsection{Classic MRTA Problem in Factory Automation}

Classical MRTA problem has been well discussed in $[65,66]$. By defining a team of robots, a set of tasks, and a collection of robots' utility, the objective of problem is to find a solution that assign tasks to robots.

By the taxonomy in [65], the MRTA problem in factory automation is ST-SR-IA optimal assignment problem. The problem can be described as follows:

Given $m$ robots and $n$ tasks, each robot is capable of executing a job, the goal is to assign robots to jobs so as to maximize overall expected utility $U$ of the system.

\subsection{Temporal-Spatial Modeling}

In factory automation, the assembly jobs for a production follow a certain sequence, a job can be executed by the assigned robot only after another assigned robot which means temporal constrain to the MRTA problem in factory automation. The production process in the factory then requires a certain flow for each certain product. Then the production lines are set up for some certain products once the production plan was made for mass production in traditional factory automation. The production line are usually fixed after testing till the production plan is finished or some break down happens. To realize the flexible production in smart factory, the production lines are able to be reconfigured quickly and the production flow can be adjusted according to the dynamic change production plans.

Consider a smart factory with $M$ types of production robots for $M$ different jobs. Each type robots dedicates to one different job. There are $N_{m}$ type- $m$ robots, $m=1,2, \ldots, M$, which are represented as $R_{m, n}, n=$ $1,2, \ldots, N_{m}$. As Figure 4 depicts the geometric arrangement, cost of transportation by AGVs for the production can be determined. $\omega_{i, j}$ is the real-time multiplexing capability of a production robot.

\subsection{Flexible MRTA Problem in Industry 4.0}

Recently, there were some research on the optimization of production suggest different objectives, such like the energy efficiency, in the MRTA problem in 


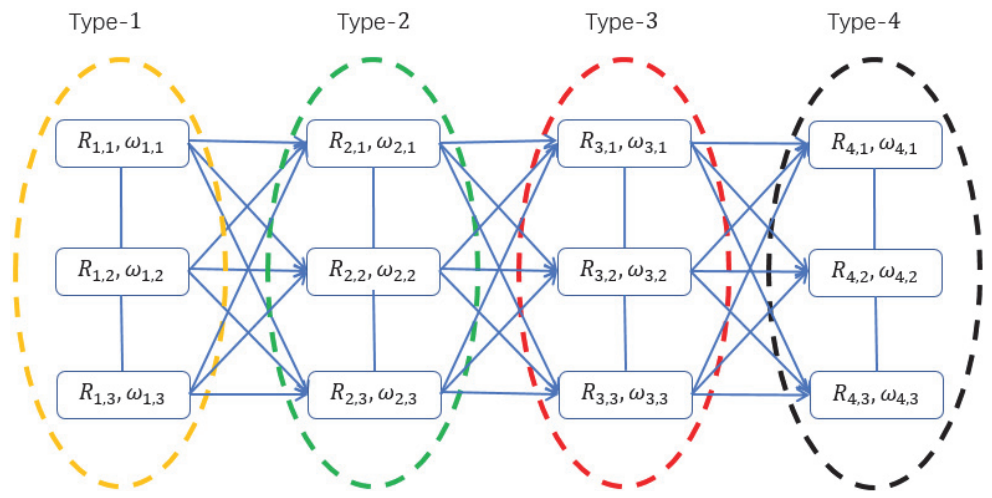

Figure 4 A four types of robots example of temporal-spatial model for MRS in a smart factory: each one production flow can be represented as a path from type- 1 robot(s) to type- 4 $\operatorname{robot}(\mathrm{s})$.

the factory [67-69]. The flexible MRTA problem in the smart factory will deal with production robots and AGVs as well. This problem must consider the production throughput and energy cost at the same time.

Base on the above statement about a smart factory, suppose there are a production plan of jobs $J=\left\{J_{k}, 1 \leq k \leq n\right\}$ to be executed over a finite horizon of time. Suppose each job is finished by a production robot in a certain period of time, we model the horizon of time as $T$ time slots. The production in Industry 4.0 can be formulated as a flexible MRTA problem with temporal and spatial constraints state above.

Consider the throughput is the first objective of production in factory. For maximizing the throughput of factory, each robots will be assigned and finished a job successfully in every time slot base on its capability and the requirement of jobs. Due to the dynamic job arrival, the flexible MRTA problem can be a sequential optimization under uncertainty and coordinating multirobot decisions [70]. The profit of robot $R_{i, j}$ finished a job is represented as $p_{i, j}$. The energy to deliver an unfinished product from robot $R_{i}$ to robot $R_{j}$ is defined as $e_{i, j}$ which relates to the physical positions of robots. The utility of robot $R_{i, j}$ finished a job is

$$
u_{i, j}=p_{i, j}-e_{i, j}
$$

The objective of the problem is find a policy that maximize the expected cumulative utility of the system. An allocation policy $X$ is a mapping from the robots to the jobs and their respective arrival time $X: J \times T \rightarrow R$. The 
utility of MRS on a policy $X_{t}$ is

$$
U_{t}=\sum_{i=1}^{M} \sum_{j=1}^{N_{i}} x_{t}^{i, j} u_{i, j}, x_{t}^{i, j} \in X_{t}
$$

The policy $X_{t}=\left\{x_{t}^{i, j}, 1 \leq t \leq T,\right\}, x_{t}^{i, j}$ is an indicator which is defined by

$$
x_{t}^{i, j}= \begin{cases}1, & \text { if } R_{i, j} \text { is assigned to a job at time } t \\ 0, & \text { otherwise }\end{cases}
$$

The optimization of real-time MRTA problem is

$$
\underset{X_{t} \in X}{\arg \max } E\left[\sum_{1 \leq t \leq T} X_{t} \cdot U_{t}\right]
$$

subject to the temporal and spatial constraints.

$$
\begin{gathered}
\sum_{i=1}^{M} \sum_{j=1}^{N_{i}} x_{t}^{i, j} \leq \omega_{i, j}, \forall t \in[1, T] \\
\sum_{t=1}^{T} \sum_{i=1}^{M} \sum_{j=1}^{N_{i}} x_{t}^{i, j} \leq n \\
x_{t}^{i, j} \in\{0,1\}, 1 \leq i \leq M, 1 \leq j \leq N_{i}, 1 \leq t \leq T
\end{gathered}
$$

\subsection{AGVs as Multiple Traveling Salesmen Problem}

The AGVs deliver the semi-products along the production flow can be formulated as the multiple traveling salesman problem (mTSP). A AGV is regarded as a salesman and the production robots are corresponding to the cities. An $\mathrm{AGV}$ follow the sequence of the production flow and visit the production one after one till the production is finished and return to the start point of the production flow. Suppose the salesmen must cover all available nodes and return to the starting nodes. Given a graph $G=(V, E)$, where $V$ is the set of $n$ nodes (tasks) and $E$ is the set of edges (the directed edges to the executing order of tasks in 4). Let $W=\left[w_{j k}\right]$ be the distance matrix associated with edge $E$.

$$
a_{j k}= \begin{cases}1, & \text { if edge }(j, k) \text { is traveled in the trip } \\ 0, & \text { otherwise }\end{cases}
$$


the mTSP problem can be formulated as

$$
\operatorname{Min} \sum_{j=1}^{n} \sum_{k=1}^{n} a_{j k} w_{j k}
$$

subject to

$$
\begin{gathered}
\sum_{k=2}^{n} a_{1 k}=m \\
\sum_{j=1}^{n} a_{j k}=1, k=2, \ldots, n \\
\sum_{k=1}^{n} a_{j k}=1, j=2, \ldots, n \\
a_{j k} \in\{0,1\}, \forall(j, k) \in E \\
\sum_{j \in S} \sum_{k \in S} a_{j k} \leq \mid \text { subTrip } \mid-1, \forall S \subseteq V-\{1\}, \text { subTrip } \neq \Phi
\end{gathered}
$$

\subsection{Desirable Wireless Networking Enables Adaptability of MRTA}

Wireless networked MRS operating under real-time MRTA is a computing technique to achieve the purpose of controling a MRS to fulfill flexible production required by industry 4.0. For realizing the flexible production in smart factory, multi-robot systems have to be highly adaptive to support reconfigurability of production lines, real-time task assignment to production robots, and reformation of AGVs. The flexible production flow can be formed according to the dynamic of real-time MRTA in smart factory. One key component of the real-time MRTA problem is the production robots. The productions robots are responsible for autonomously execute tasks that are assigned to them. Another key component is the AGVs. The productions robots and AGVs are equipped with a large number the sensors that keep updating their status. During the process, the production robots tasks assignment are executed by the edge server, the recofiguration of production robot can be done through the cloud service. The AGVs using sensor network obviously will be wireless connected in real-time manner. The edge services could be useful to improve the decisions making during the period. 
The real-time MRTA is a one-shot decision of the system that means the timing constraint and correctness of communications are highly required for communication. The requirement on the delay and reliability are more important than others. In order to effectively communicate with other robots in the system, the robots must capture the data in time to make their decision. Since the mobile devices are supplied with battery which capacities are limited, the energy efficiency is also very important in the smart factory. Therefore, as indicated in [71] and table 1, MRTA bypasses real-time motion control by way of task assignment, the required latency is $10-50 \mathrm{~ms}$. Consider a MRS under Robot-to-Infrastructure (R2I) communication, a typical 5G microcell serves $200 \mathrm{UEs}$ [72], with average round-trip-time (RTT) of $\tau$. Assume 100 out of $200 U E s$ are robots, other $U E s$ can be other devices in network such as IoT devices, and reliability $10^{-3}[11,18,64]$, the $\tau$ should be $0.099-0.495 \mathrm{~ms}$, which is smaller than current state-of-art $5 \mathrm{G}$ performance metrics.

\section{Collaborative Multi-robot Systems}

The artificial intelligence (AI) integrate with robotics has been a new technology paradigm for the smart factory $[4,68]$. In order to adapt quickly to the customized production, the multi-agent system could be a solution to the challenge [44,73]. [74] proposed an example of smart factory in which human workers and robots are agents that worked together to be assigned production job. And the agents are capable of making decision [42] presented an architecture of AI-driven smart factory that also showed the flexible manufacturing line can be achieved by multi-agent systems. It can perceive the dynamic of the environment and adapt to the external needs with network collaboration.

\subsection{Robots Using Al}

With the development of manufacturing technologies and processes, manufacturing tools have been designed to integrate various operations. Greater integration of processes have been applied to enhance factory automation. Sensors and software capabilities are making the new manufacturing robots and transportation robots smarter $[5,75]$.

Reinforcement learning (RL) is widely applied to represent the dynamic behavior of a smart agent interacting with environment and/or other agents. Agent, reward, and environment are the three essential elements of RL. At 
any time $t$, the agent interacts with environment and determines the state $s_{t}$ of the environment with sensors. The environment provides a reward $r_{t}$ to the agent when the agent takes an action $a_{t}$ according to the policy $\pi$. Then the system transit to next state $s_{t+1}$. RL is expected to derive an optimal strategy for a series of actions for the robot according to the state of the system and the reward for the actions. Let $V(t)$ be the state-action value function, the learning is represented by

$$
Q\left(s_{t}, a_{t}\right)=\mathbb{E}\left[r_{t+1}\right]+\gamma \sum_{s_{t+1}} P\left(s_{t+1} \mid s_{t}, a_{t}\right) V\left(s_{t+1}\right)
$$

where $\gamma$ is the discount parameter, $0 \leq \gamma \leq 1$.

However, the Markov property does not necessarily hold for observations of the robots. For example, the AGVs in the smart factory work independently, the environment is partially observable thus the observation of each robot is different. The agent could to calculate the most likely state and take an action accordingly. In order to maintain the Markov process, the agent keeps an internal belief state $b_{i}$ which summarizes the robot's own experience. The agent estimates the current state and updates the belief state $b_{i+1}$ based on last action $a_{i}$, current action $a_{i+1}$, previous belief state $b_{i}$. Given initial belief state and the past observation-action history of the agent, the belief state has a probability distribution over the states of the system. The learning spirit can be updated by the belief of robot as follows

$$
Q\left(b_{t}, a_{t}\right)=\mathbb{E}\left[r_{t+1}\right]+\gamma \sum_{b_{t+1}} P\left(b_{t+1} \mid b_{t}, a_{t}\right) V\left(b_{t+1}\right)
$$

where $\gamma$ is the discount parameter, $0 \leq \gamma \leq 1$.

\subsection{Wireless Communication Enhances Performance of Multi-Robot System}

The multi-agent RL extends the application area of AI and also make the decision making to more complex problem. In the smart factory, the AGVs that work together to realize the intelligent transportation for the flexible MRTA can be regarded as a multi-robot system [47] apply Q-learning to model AGVs transporting the semi-production scenario in the smart factory that each agent behaves in reinforcement learning. An AGV can be viewed as an agent of RL to the optimal policy toward the position of next production robot. The agent only knows its destination on the streets map but does not 


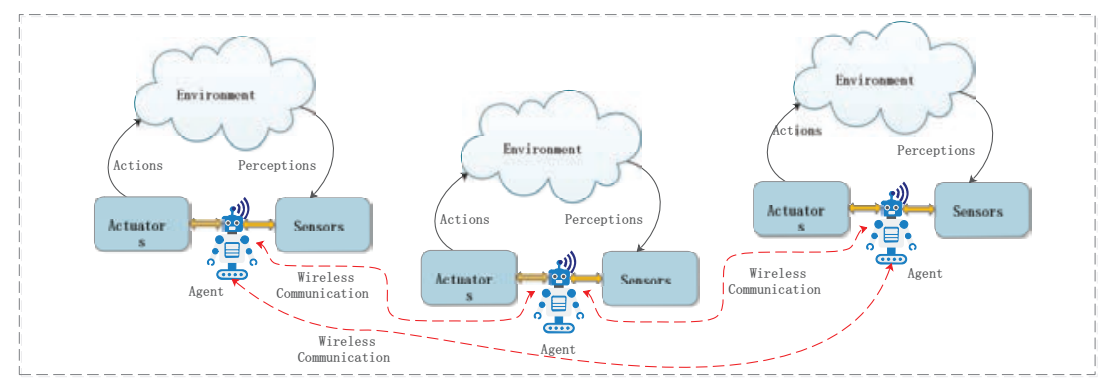

Figure 5 Wireless communication in multi-robot system.

know others on the streets. A modification of slotted ALOHA named as realtime ALOHA is proposed to support RL for the multi-robot system. The experiments show that the whole performance of AGVs system are greatly enhanced by the assistance of communication for RL.

[76] studied another critical scenario of MAS system in the smart factory. The autonomous robots were governed by RL and navigated on Manhattan streets, following the rule of stop sign in the intersections. The robots shared the useful information (i.e. reward map and private reference) with each other and thus improve the average delay of the trips [54] studied a collaborative multi-agent system in which each agent can only partially observe the environment and other agents. The agents learn an optimal policy based on the believes of the states. Multi-agents of RL collaborate by wireless communication can obviously improve their collective performance. As Figure 5 shows, the collaborative robots communicate with each other to finish the product processes. Further realistic situations in wireless communications, like the random errors and multiple access communication among AI agents also had been studied. The paper also shows that wireless robotic communication reveals many new opportunities for robotics, multi-agent systems.

\subsection{Communication Requirements for Smart Factory}

The vision of smart factory is built on the idea of fully automated industry that can reduce human workforce and improve efficiency while achieve an energysaving way at the same time. Industrial robots have been widely deployed in the factories. It is self-evident that the operation of the MRS need to sense and actuate the robots. The multi-robot systems form by the interconnected production robots and AGVs in the smart factory raise the communication requirements not only on the reliability but also the flexibility of rotation and mobility of devices [77]. 
Most of the traditional industrial communications are based on wired technologies. The industrial networks can offer a fast and reliable connection with hundreds of nodes in wide areas [10]. The most critical profiles of the IEC 61850 for motion control are generic object-oriented substation event (GOOSE) and sampled values (SV) which can achieved a minimum end-toend latency $0.3 \mathrm{~ms}$ for a size of 100 nodes network [78]. While the wireless technologies have been developed in the past two decades for industrial application with the features of the lower cabling cost, better devices mobility, and easier configuration [79]. The IEEE 802.15.4 [80] is a popular standard for the wireless industrial network based on which several industry wireless networking solution have been developed. The Industrial wireless sensor networks (IWSNs) [81], WirelessHART and ISA 100.11a [82] use a timeslotted channel-hopping medium access control layer with a minimum of $10 \mathrm{~ms}$ duration which is only good enough for industrial processes monitoring due to the limitations of the IEEE 802.15.1 standards [34]. IEEE 802.11 WiFi [83] has the capability of using multiple-user transmissions simultaneously to achieve very high data rates to improve control latency and reliability. One of the improved solutions is the fifth 5G mobile networks that develop uRLLC for factory automation that is able to offer the end-to-end latency below $1 \mathrm{~ms}$ for a wide range of applications.

However, current version of $5 \mathrm{G}$ can not satisfy the real-time requirements of the factory automation [84]. In the scenario of smart factory, the traffic between agents are reward maps and policies. There are also sensors data be collected for deep learning and data fusion. The average traffic of AGVs and production robots could be up to $1000 \mathrm{Mbps}$. For closed loop regulatory control for the production robots, the reliability is suggested $99.999 \%-99.99999 \%$ and the latency is 2-10 $\mathrm{ms}$. Assuming the AGVs moving with a speed of $2 \mathrm{~m} / \mathrm{s}$, the suggested reliability is $99.999 \%$ $99.9999 \%$ and the latency is $5-10 \mathrm{~ms}$ [13,31,33,48]. None of the current wireless network solutions can support the advanced requirements to the ultralow latency and ultrareliability communications in smart factory. However, resilient collaborative MRS that is under investigations may alleviate the requirement of reliability and thus relieve the strict requirement of wireless networking.

\section{Resilient Cyber-physical Multi-robot Systems}

The robots in the multi-robot system act together in manufacturing processes. The cyber network consisting of autonomous agents is organized 
in accordance with task that is assigned by a corresponding portfolio of products $[4,39,85]$. However, there are uncertainties in the environment, measurement, and estimation, etc.. The main challenge for the system is capability to react to the faults in the dynamical environment $[39,86,87]$. Due to the lack of global knowledge of the of environment, the smart factory can adopt the social learning scheme take the advantage of the wireless networking to control the robots.

\subsection{Sequential Actions in MAS}

In the smart factory, multi-agent system can be viewed as the coevolution of cyber-network and physical-network. Each individual agent can work on the self-determined tasks while obtain other agents' information from observation and communication. All agents act collaboratively toward accomplishing the production job. Agents' decisions reflect the correspondent physical dynamics in the system.

Consider a smart factory in which there are several production lines and a number of production robots on each production line. Every robot has different function dedicating to special physical processes. For a production robot, a manufacturing task is to take some actions at a particular precision scale of slots that can be represent as scaled sequential decision. The agents can induce sequential dependency in the accumulate error to reduce the effects from the failure.

\subsection{Social Learning Model}

In a MAS in smart factory, the cyber-domain deals with decision, coordination, control and optimization through communication, networking, and observations between agents, the physical-domain deals with interaction of each agent within the physical world. By decision-theoretic modeling, smart agents that make decisions not only perform physical measurement of the state of system but also consider information obtained form the cyber network of agents. The collective actions and behavior of the networked agents can possibly influence the physical states thus the cyber and physical interaction in MAS.

The MRS can be represented as a set of state space $\mathcal{S}$. Each state $s \in$ $\mathcal{S}$ is associated with the prior probability $p(s)$. There is an action space $\mathcal{A}$ associated with each state. A utility function $U: \mathcal{S} \times \mathcal{A} \rightarrow \mathbb{R}$ maps the stateaction pair $(s, a), s \in \mathcal{S}, a \in \mathcal{A}$ to a real number $U(s, a) \in \mathbb{R}$. A single agent $i$ makes a measurement $X_{i}$ to the state of world $s$. Given the measurement 
$X_{i}=x_{i}$, the decision to take action $a_{i}$ to maximize utility which is calculated by $\mathbb{E}\left[U_{i}\left(s_{i}, a_{i}\right) \mid X_{i}=x_{i}, y_{i}\right]$.

The cyber and physical interaction plays a vital role in the decision model of MAS. Suppose the network topology of agents can be presented by a graph $G(N, E)$, where $N=1,2, \ldots, n$ is a finite agents set and $E=$ $\{(i, j) \mid i$ has a link toward $j\}$ is the set of edge. Let the social observation $\mathcal{Y}_{i}=\left\{a_{j} \mid(j, i) \in E\right\}$ represents decisions set collects from local network neighborhood. The dependence of utility function on social observation set is $p_{i}\left(s_{i} \mid \mathcal{Y}_{i}\right)$. The dependence of utility function on observation set is $U\left(s_{i}, a_{i} ; \mathcal{Y}_{i}\right)$. Suppose the MAS operates over a finite-horizon of time steps $t=1,2, \ldots T$. At each time step $t$, every agent $i \in N$ gets a measurement $X_{i}^{t}=x_{i}^{t}$. From the distribution $f_{X_{i}}^{t}\left(x_{i}{ }^{t} \mid s_{i}^{t}\right)$ and observation set $\mathcal{Y}_{i}^{t}$. The agent makes a decision for expected utility

$$
a_{i}^{t}=\underset{a}{\arg \max } \mathbb{E}\left[U_{i}\left(s_{i}^{t}, a\right) \mid X_{i}^{t}=x_{i}^{t}, \mathcal{Y}_{i}^{t}\right]
$$

At time $t$ the system can be defined by a performance function $J^{t}$ that maps the outcome to a real number by

$$
J^{t}\left(\left\{s_{i}^{t}\right\}_{i=1}^{n},\left\{a_{i}^{t}\right\}_{i=1}^{n}\right) \in \mathbb{R}
$$

\subsection{Network Topology for Interactive MAS}

When a robot is executing planed actions, it could be beneficial to receive information from other robot to improve safety and reduce errors. Since the limit of computing power and other resources, The computation will partly or entirely load to edge devices or cloud services in the data centers [88]. At the same time, with the aids of deep learning, the agent will have better decisions quickly. The whole decision making must be happened by wireless communication.

In smart factory, smart agents in the MAS exchange cascade information, such as state and policy, with other interacting agents in the same production flow so as to reduce accumulative error thus leverage the resilience of smart factory.

Experiments show that network topology plays a vital role to the performance of the system. In an ideal situation, fully connected agents can have complete synchronization with each other, the MAS has the best sequential calibration behavior. However, fully connected topology is not necessarily good since the errors from communication links will bring in more noise into decision of the agents and significantly degrade the gain from wireless 
networking. The small-world networks [4,54] is a good way to enhance information dissemination and lead to a resilient smart factory to errors caused by robots and wireless networking. A flexible topology for the collaborative cyber-physical system is desired.

The closed-loop control applications typically demand a cycle time of 2-10 ms. In the smart manufacturing scenario, the cyber-physical multirobot system requires the wireless network a cycle time of $0.5-2 \mathrm{~ms}$ with reliability of $99.9999 \%-99.99999 \%[13,31]$.

\section{Cyber Security in Smart Factory}

In addition to the reliability issues discussed above due to the MRSs in smart factories themselves, MRSs of smart factories enabling $6 \mathrm{G}$ wireless networks are members of the cyber physical system, they would be exposed to security threats from complex cyberspace malicious attacks and from their own vulnerabilities [89].

$[90,91]$ discussed the research status on the security of smart manufacturing $[92,93]$ presented the security issues in the MRS as a cyberphysical system. Other attack to sensing capability and physical tools, etc. that would cause systematic cascading was also discussed in [94,95]. [96] presented the attacks and defenses about deep neural network. Undoubtedly, security issues related to Industry 4.0 are inevitable and very important research topics.

The security of the smart factory focuses on discussing the confidentiality, integrity, availability, authenticity, and controllability [97, 98]. An overall comprehending of the security issues faced by smart factories is critical to guaranteeing the security of them. In this section, the security threats and attack technologies about smart factories are introduced from a holistic hierarchical perspective.

According to the defining cyberspace security [99], combined with the characteristics of $6 \mathrm{G}$ enabled wireless networking smart factories, a cyberspace security framework is set up for smart factories in Figure 6. From a spatial view, the smart factory security framework is divided into five layers, device layer security, communication layer security, system layer security, data layer security and application layer security.

The device layer security concerns physical security, consisting of devices and hardware security, device safety, and environmental safety. The communication layer security refers to wireless networking-related security, including protocol security, transfer security, access security, etc. The system 


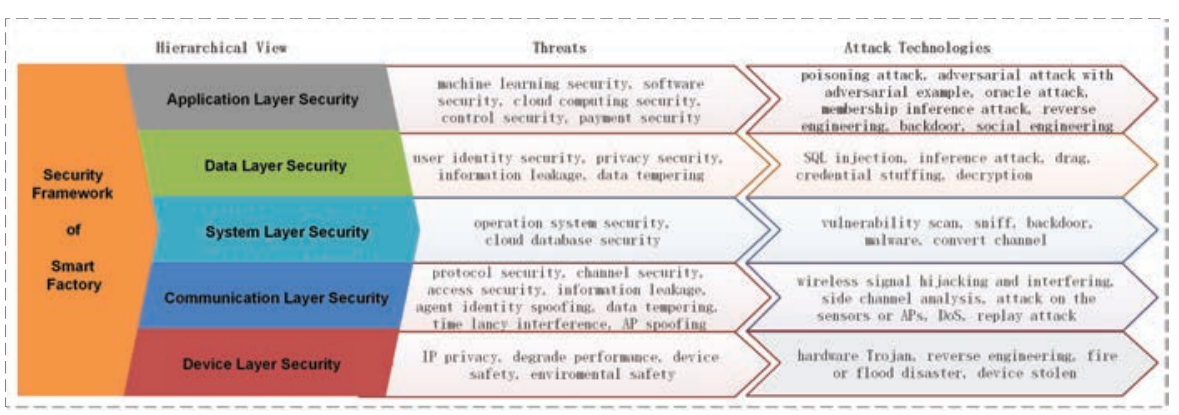

Figure 6 Security framework of $6 \mathrm{G}$ enabled smart factory.

layer security means system-level-related security, containing operating system security, database security. The data layer security is the data-related security, and identity security and privacy security are mainly concerned. The application layer security is considered the information application-related security, content security, machine learning security, payment security, and control security are involved.

According to the attributes, each layer faces its own security threats. The threats cover data tampering, Intellectual property (IP) piracy, privacy protection [98], information leakage, degraded performance, denial of service (DoS) [100], social engineering [97], machine learning security [96], identity spoofing (including collaborative MRS robots' identity and human users' identity) [101], latency interference [98], and so forth.

To implement the malicious behaviors, attack technologies can be wireless signal hijacking inerfering, hardware Trojans [102], malware, side channel analysis [97], SQL injection, attack on the sensors and APs [97], poisoning attack for machine learning, adversarial attack with adversarial example, oracle attack, membership inference attack, and reverse engineering [96], to name a few.

The smart factories should consider a wide range of attacks, and the security architecture should be set up to protect them [72]. At the same time, the security architecture must be meet the communication requirements of $6 \mathrm{G}$, such as uRLLC and mMTC. Furthermore, it is worth to note that for the 6G enabled wireless networks smart factory, the security issues from MRS collaborative communication [103], and from machine learning [96] should be paid enough attention to. 


\section{Conclusion}

The main trend of the smart factories is realizing flexible production according to the dynamic demand from the market. Take the advantage of wireless networking technology, the smart factory has brought huge opportunities and challenges to wireless network technology. This paper presents a vision of a smart factory as a use case for $6 \mathrm{G}$ wireless communication, mainly introduce the smart factory as wireless networked multi-robot systems to realize customization with the support of collective intelligence and mobile intelligence. what's more, real-time MRTA, collaborative and resilient MRS, and security issues are discussed. We hope this paper can establish a 6G bled smart factory profile for the readers, and promote related research on $6 \mathrm{G}$ enabled smart factories.

\section{References}

[1] S. Y. Lien, K. C. Chen, and Y. H. Lin. Toward ubiquitous massive accesses in $3 \mathrm{GPP}$ machine-to-machine communications. IEEE Communications Magazine, 49(4):66-74, 2011.

[2] S.Y. Lien and K.C. Chen. Massive access management for QoS guarantees in 3GPP machine-to-machine communications. IEEE Communications Letters, 15(3):311-313, 2011.

[3] K. C. Chen, T. Zhang, R. D. Gitlin, and G. Fettweis. Ultra-low latency mobile networking. IEEE Network, 33(2):181-187, 2019.

[4] K. C. Chen, S. C. Lin, J. H. Hsiao, C. H. Liu, A. F. Molisch, and G. P. Fettweis. Wireless networked multirobot systems in smart factories. Proceedings of the IEEE, 109(4):468-494, 2021.

[5] A. Kusiak. Smart manufacturing. International Journal of Production Research, 56(1-2):508-517, 2018.

[6] A. Grau, M. Indri, L. Bello, and T.O. Sauter. Robots in industry. IEEE Industrial Electronics, 15(1 (Supplement)):50-61, 2021.

[7] C. Aracil, G. Aziebig, P. Korondi, S. Oh, Z Tan, M. Ruderman, W. He, L. Ding, H. Luo, S. Yin, and A. Haghani. Toward smart systems. IEEE Industrial Electronics, 15(1 (Supplement)):104-114, 2021.

[8] A. Ahmadi, M. Moradi, C. Cherifi, V. Cheutet, and Y. Ouzrout. Wireless connectivity of CPS for smart manufacturing: A survey. In 2018 12th International Conference on Software, Knowledge, Information Management Applications (SKIMA), pages 1-8, 2018. 
[9] D. Baumann, F. Mager, U. Wetzker, L. Thiele, M. Zimmerling, and S. Trimpe. Wireless control for smart manufacturing: Recent approaches and open challenges. Proceedings of the IEEE, 109(4):441-467, 2021.

[10] S. Vitturi, C. Zunino, and T. Sauter. Industrial communication systems and their future challenges: Next-generation ethernet, IIoT, and 5G. Proceedings of the IEEE, 107(6):944-961, 2019.

[11] I. F. Akyildiz, A. Kak, and S. Nie. 6G and beyond: The future of wireless communications systems. IEEE Access, 8:133995-134030, 2020.

[12] J. H. Hsiao and K. C. Chen. Network analysis of collaborative cyberphysical multi-agent smart manufacturing systems: Invited paper. In 2019 IEEE/CIC International Conference on Communications in China (ICCC), pages 1-6, 2019.

[13] M. Giordani, M. Polese, M. Mezzavilla, S. Rangan, and M. Zorzi. Toward 6G networks: Use cases and technologies. IEEE Communications Magazine, 58(3):55-61, 2020.

[14] C. She, C. Sun, Z. Gu, Y. Li, C. Yang, H. V. Poor, and B. Vucetic. A tutorial on ultrareliable and low-latency communications in 6G: Integrating domain knowledge into deep learning. Proceedings of the IEEE, 109(3):204-246, 2021.

[15] Y. K. Liu, M. Kashef, K. B Lee, L. Benmohamed, and R. Candell. Wireless network design for emerging IIoT applications: reference framework and use cases. Proceedings of the IEEE, 107(6):1166-1192, 2019.

[16] S. Mumtaz, A. Bo, A. Al-Dulaimi, and K. Tsang. Guest editorial $5 \mathrm{G}$ and beyond mobile technologies and applications for industrial IoT (IIoT). IEEE Transactions on Industrial Informatics, 14(6):25882591, 2018.

[17] D. Bruckner, M. Stănică, R. Blair, S. Schriegel, S. Kehrer, M. Seewald, and T. Sauter. An introduction to opc ua tsn for industrial communication systems. Proceedings of the IEEE, 107(6):1121-1131, 2019.

[18] K. B. Letaief, W. Chen, Y. Shi, J. Zhang, and Y. A. Zhang. The roadmap to 6G: Ai empowered wireless networks. IEEE Communications Magazine, 57(8):84-90, 2019.

[19] S. Vitturi, T. Sauter, and Z. B. Pang. Real-time networks and protocols for factory automation and process control systems [scanning the issue]. Proceedings of the IEEE, 107(6):939-943, 2019. 
[20] S. Y. Lien, T. H. Liau, C. Y. Kao, and K. C. Chen. Cooperative access class barring for machine-to-machine communications. IEEE Transactions on Wireless Communications, 11(1):27-32, 2012.

[21] X. Chen, D. W. K. Ng, W. Yu, E. G. Larsson, N. Al-Dhahir, and R. Schober. Massive access for 5G and beyond. IEEE Journal on Selected Areas in Communications, 39(3):615-637, 2021.

[22] A. Al-Fuqaha, M. Guizani, M. Mohammadi, M. Aledhari, and M. Ayyash. Internet of things: A survey on enabling technologies, protocols, and applications. IEEE communications surveys \& tutorials, 17(4):2347-2376, 2015.

[23] M. Agiwal, A. Roy, and N. Saxena. Next generation 5G wireless networks: A comprehensive survey. IEEE Communications Surveys \& Tutorials, 18(3):1617-1655, 2016.

[24] Z. H. Li, J. Chen, R. Ni, S. Chen, X. Li, and Q. Y. Zhao. Enabling heterogeneous MMTC by energy-efficient and connectivity-aware clustering and routing. In 2017 IEEE Globecom Workshops (GC Wkshps), pages 1-6. IEEE, 2017.

[25] P. Popovski, K. F. Trillingsgaard, O. Simeone, and G. Durisi. 5G wireless network slicing for eMBB, URLLC, and mMTC: A communication-theoretic view. IEEE Access, 6:55765-55779, 2018.

[26] B. A. Jayawickrama, Y. He, E. Dutkiewicz, and M. D. Mueck. Scalable spectrum access system for massive machine type communication. IEEE Network, 32(3):154-160, 2018.

[27] C. Bockelmann, N. K Pratas, G. Wunder, S. Saur, M. Navarro, D. Gregoratti, G. Vivier, E. De Carvalho, Y. L. Ji, Č. Stefanović, et al. Towards massive connectivity support for scalable mMTC communications in 5G networks. IEEE access, 6:28969-28992, 2018.

[28] P. Castagno, V. Mancuso, M. Sereno, and M. A. Marsan. A simple model of mtc in smart factories. In IEEE INFOCOM 2018 - IEEE Conference on Computer Communications, pages 2591-2599, 2018.

[29] T. M. Ho, T. D. Tran, T. T. Nguyen, S.M. Kazmi, L. B. Le, C. S. Hong, and L. Hanzo. Next-generation wireless solutions for the smart factory, smart vehicles, the smart grid and smart cities. arXiv preprint arXiv:1907.10102, 2019.

[30] G. P. Fettweis. The tactile internet: Applications and challenges. IEEE Vehicular Technology Magazine, 9(1):64-70, 2014.

[31] A. Aijaz and M. Sooriyabandara. The tactile internet for industries: A review. Proceedings of the IEEE, 107(2):414-435, 2019. 
[32] W. Haerick and M. Gupta. $5 \mathrm{G}$ and the factories of the future. 5G-PPP White Paper, 2015.

[33] M. Luvisotto, Z. B. Pang, and D. Dzung. Ultra high performance wireless control for critical applications: Challenges and directions. IEEE Transactions on Industrial Informatics, 13(3):1448-1459, 2016.

[34] M. Luvisotto, Z. B. Pang, and D. Dzung. High-performance wireless networks for industrial control applications: New targets and feasibility. Proceedings of the IEEE, 107(6):1074-1093, 2019.

[35] T. Hößler, M. Simsek, and G. P Fettweis. Mission reliability for urllc in wireless networks. IEEE Communications Letters, 22(11):2350-2353, 2018.

[36] M. Klügel, M. Mamduhi, O. Ayan, M. Vilgelm, K. H Johansson, $\mathrm{S}$. Hirche, and W. Kellerer. Joint cross-layer optimization in real-time networked control systems. IEEE Transactions on Control of Network Systems, 7(4):1903-1915, 2020.

[37] Q. B. Jin, S. Wu, R. D. Zhang, and R. Q. Lu. Improved minmax control for industrial networked systems over imperfect communication. IEEE Transactions on Systems, Man, and Cybernetics: Systems, 50(4):13101317, 2017.

[38] K. S. Kim, D. K. Kim, C. B. Chae, S. Choi, Y. Chai Ko, J. Kim, Y. G. Lim, M. Yang, S. Kim, B. Lim, et al. Ultrareliable and low-latency communication techniques for tactile internet services. Proceedings of the IEEE, 107(2):376-393, 2018.

[39] D. Ratasich, F. Khalid, F. Geissler, R. Grosu, M. Shafique, and E. Bartocci. A roadmap toward the resilient internet of things for cyber-physical systems. IEEE Access, 7:13260-13283, 2019.

[40] S. Ji, S. Lee, S. Yoo, I. Suh, I. Kwon, F. C. Park, S. Lee, and H. Kim. Learning-based automation of robotic assembly for smart manufacturing. Proceedings of the IEEE, 109(4):423-440, 2021.

[41] B. Chen, J. Wan, L. Shu, P. Li, M. Mukherjee, and B. Yin. Smart factory of industry 4.0: Key technologies, application case, and challenges. IEEE Access, 6:6505-6519, 2018.

[42] J. Wan, X. Li, H. N. Dai, A. Kusiak, M. Martínez-García, and D. Li. Artificial-intelligence-driven customized manufacturing factory: Key technologies, applications, and challenges. Proceedings of the IEEE, 109(4):377-398, 2021. 
[43] W. M. Shen, L. H. Wang, and Q. Hao. Agent-based distributed manufacturing process planning and scheduling: a state-of-the-art survey. IEEE Transactions on Systems, Man, and Cybernetics, Part $C$ (Applications and Reviews), 36(4):563-577, 2006.

[44] A. Giret, D. Trentesaux, M. A. Salido, E. Garcia, and E. Adam. A holonic multi-agent methodology to design sustainable intelligent manufacturing control systems. Journal of cleaner production, 167:1370-1386, 2017.

[45] Y. Liu and X. Xu. Industry 4.0 and cloud manufacturing: A comparative analysis. Journal of Manufacturing Science and Engineering, 139(3):034701, 2017.

[46] D. A. Chekired, L. Khoukhi, and H. T. Mouftah. Industrial IoT data scheduling based on hierarchical fog computing: A key for enabling smart factory. IEEE Transactions on Industrial Informatics, 14(10):4590-4602, 2018.

[47] E. Ko, K. C. Chen, and S. Y. Lien. Collaborative partially-observable reinforcement learning using wireless communications. IEEE ICC, 00(1):1-6, 2021.

[48] W. Liang, M. Zheng, J. Zhang, H. Shi, H. Yu, Y. Yang, S. Liu, W. Yang, and X. Zhao. Wia-fa and its applications to digital factory: A wireless network solution for factory automation. Proceedings of the IEEE, 107(6):1053-1073, 2019.

[49] Y. F. Zhang, C. Qian, J. X. Lv, and Y. Liu. Agent and cyber-physical system based self-organizing and self-adaptive intelligent shopfloor. IEEE Transactions on Industrial Informatics, 13(2):737-747, 2016.

[50] Y. F. Zhang, Z. F. Zhu, and J. X. Lv. CPS-based smart control model for shopfloor material handling. IEEE Transactions on Industrial Informatics, 14(4):1764-1775, 2017.

[51] E. A Oyekanlu, A. C Smith, W. P Thomas, G. Mulroy, D. Hitesh, M. Ramsey, D. J Kuhn, J. D Mcghinnis, S. C Buonavita, N. A Looper, et al. A review of recent advances in automated guided vehicle technologies: Integration challenges and research areas for 5G-based smart manufacturing applications. IEEE Access, 8:202312-202353, 2020.

[52] Y. Luo, Y. Duan, W. F. Li, P. Pace, and G. Fortino. A novel mobile and hierarchical data transmission architecture for smart factories. IEEE Transactions on Industrial Informatics, 14(8):3534-3546, 2018.

[53] G. Fortino, F. Messina, D. Rosaci, G. M. L. Sarné, and C. Savaglio. A trust-based team formation framework for mobile intelligence in smart 
factories. IEEE Transactions on Industrial Informatics, 16(9):61336142, 2020.

[54] K. C. Chen and H. M. Hung. Wireless robotic communication for collaborative multi-agent systems. In ICC 2019 - 2019 IEEE International Conference on Communications (ICC), pages 1-7, 2019.

[55] I. Álvarez, A. Ballesteros, M. Barranco, D. Gessner, S. Djerasevic, and J. Proenza. Fault tolerance in highly reliable ethernet-based industrial systems. Proceedings of the IEEE, 107(6):977-1010, 2019.

[56] J. Theunissen, H. Xu, R. Y. Zhong, and X. Xu. Smart agv system for manufacturing shopfloor in the context of industry 4.0. In 2018 25th International Conference on Mechatronics and Machine Vision in Practice (M2VIP), pages 1-6, 2018.

[57] G. Berardinelli, N.H. Mahmood, I. Rodriguez, and P. Mogensen. Beyond 5g wireless irt for industry 4.0: Design principles and spectrum aspects. In 2018 IEEE Globecom Workshops (GC Wkshps), pages 1-6, 2018.

[58] I. Jovović, S. Husnjak, I. Forenbacher, and S. Maček. Innovative application of $5 \mathrm{G}$ and blockchain technology in industry 4.0. EAI Endorsed Transactions on Industrial Networks and Intelligent Systems, 6(18), 2019.

[59] K. B. Letaief, W. Chen, Y. Shi, J. Zhang, and Y. A. Zhang. The roadmap to 6G: Ai empowered wireless networks. IEEE Communications Magazine, 57(8):84-90, 2019.

[60] International Telecommunication Union (ITU). Minimum requirements related to technical performance for imt-2020 radio interface (s). Radiocommunication Sector of ITU - ITU-R, pages 1-9, 2017.

[61] J.Walia, H.Hämmäinen, K.Kilkki, and S.Yrjölä. 5G network slicing strategies for a smart factory. Computers in industry, 111:108-120, 2019.

[62] D. Cavalcanti, J. Perez-Ramirez, M. M. Rashid, J. Fang, M. Galeev, and K. B. Stanton. Extending accurate time distribution and timeliness capabilities over the air to enable future wireless industrial automation systems. Proceedings of the IEEE, 107(6):1132-1152, 2019.

[63] M. Felser, M. Rentschler, and O. Kleineberg. Coexistence standardization of operation technology and information technology. Proceedings of the IEEE, 107(6):962-976, 2019.

[64] I. F. Akyildiz, A. Kak, and S. Nie. 6G and beyond: The future of wireless communications systems. IEEE Access, 8:133995-134030, 2020. 
[65] B. P. Gerkey and M. J. Matarić. A formal analysis and taxonomy of task allocation in multi-robot systems. The International Journal of Robotics Research, 23(9):939-954, 2004.

[66] K. C. Chen. Artificial Intelligence in Wireless Robotics. River Publisher, 2020.

[67] Z. Liu, H. Wang, W. Chen, J. Yu, and J. Chen. An incidental delivery based method for resolving multirobot pairwised transportation problems. IEEE Transactions on Intelligent Transportation Systems, 17(7):1852-1866, 2016.

[68] M. Pearce, B. Mutlu, J. Shah, and R. Radwin. Optimizing makespan and ergonomics in integrating collaborative robots into manufacturing processes. IEEE Transactions on Automation Science and Engineering, 15(4):1772-1784, 2018.

[69] C. Nam and D. A. Shell. Robots in the huddle: Upfront computation to reduce global communication at run time in multirobot task allocation. IEEE Transactions on Robotics, 36(1):125-141, 2020.

[70] W.B. Powell, H. Simao, and B. Bouzaiene-Ayari. Approximate dynamic programming in transportation and logistics: a unified framework. EURO Journal on Transportation and Logistics, 1(3):237-284, 2012.

[71] X. M. Li, D. Li, J. F. Wan, A. V Vasilakos, C. F. Lai, and S. Y. Wang. A review of industrial wireless networks in the context of industry 4.0. Wireless networks, 23(1):23-41, 2017.

[72] 5G ACIA. 5G for connected industries and automation. white paper, 2nd ed. 2019. https://www.5gacia.org/publications/5g-for-connected-i ndustries-and-automation-white-paper/.

[73] U. Kannengiesser and H. Müller. Towards agent-based smart factories: A subject-oriented modeling approach. In 2013 IEEE/WIC/ACM International Joint Conferences on Web Intelligence (WI) and Intelligent Agent Technologies (IAT), volume 3, pages 83-86. IEEE, 2013.

[74] I. Kovalenko, D. Tilbury, and K. Barton. The model-based product agent: A control oriented architecture for intelligent products in multi-agent manufacturing systems. Control Engineering Practice, 86:105-117, 2019.

[75] H. Feng, J. Llorca, A. M Tulino, and A. F Molisch. Optimal dynamic cloud network control. IEEE/ACM Transactions on Networking, 26(5):2118-2131, 2018.

[76] E. Ko and K. C. Chen. Wireless communications meets artificial intelligence: An illustration by autonomous vehicles on manhattan streets. In 
2018 IEEE Global Communications Conference (GLOBECOM), pages $1-7,2018$.

[77] Xavier Vilajosana, Thomas Watteyne, Mališa Vučinić, Tengfei Chang, and Kristofer SJ Pister. 6tisch: Industrial performance for ipv6 internetof-things networks. Proceedings of the IEEE, 107(6):1153-1165, 2019.

[78] Huu-Dung Ngo and Hyo-Sik Yang. Latency and traffic reduction for process-level network in smart substation based on high-availability seamless redundancy. IEEE Transactions on Industrial Electronics, 63(4):2181-2189, 2015.

[79] Federico Tramarin, Aloysius K Mok, and Song Han. Real-time and reliable industrial control over wireless lans: Algorithms, protocols, and future directions. Proceedings of the IEEE, 107(6):1027-1052, 2019.

[80] Online. Ieee 802.15 wpans task group 4. http://www.iee802.org/15/p ub/TG4.html.

[81] Johan Åkerberg, Mikael Gidlund, and Mats Björkman. Future research challenges in wireless sensor and actuator networks targeting industrial automation. In 2011 9th IEEE International Conference on Industrial Informatics, pages 410-415. IEEE, 2011.

[82] Stig Petersen and Simon Carlsen. Wirelesshart versus isa100. 11a: The format war hits the factory floor. IEEE Industrial Electronics Magazine, 5(4):23-34, 2011.

[83] Online. Ieee p802.11— task group ax. http://www.ieee802.org/11/Rep orts/tgax_update.htm.

[84] Petar Popovski, Jimmy J Nielsen, Cedomir Stefanovic, Elisabeth De Carvalho, Erik Strom, Kasper F Trillingsgaard, Alexandru-Sabin Bana, Dong Min Kim, Radoslaw Kotaba, Jihong Park, et al. Wireless access for ultra-reliable low-latency communication: Principles and building blocks. Ieee Network, 32(2):16-23, 2018.

[85] P. J. Mosterman and J. Zander. Industry 4.0 as a cyber-physical system study. Software \& Systems Modeling, 15(1):17-29, 2016.

[86] P. Derler, E. A. Lee, and A. Sangiovanni Vincentelli. Modeling cyber-physical systems. Proceedings of the IEEE, 100(1):13-28, 2012.

[87] K. Kim and P. R. Kumar. Cyber-physical systems: A perspective at the centennial. Proceedings of the IEEE, 100(Special Centennial Issue):1287-1308, 2012. 
[88] J. Abbenseth, F.G. Lopez, C. Henkel, and S. Dörr. Cloud-based cooperative navigation for mobile service robots in dynamic industrial environments. In Proceedings of the Symposium on Applied Computing, pages 283-288, 2017.

[89] B. X. Fang. Cyberspace sovereignty. Springer, 2018.

[90] Nilufer Tuptuk and Stephen Hailes. Security of smart manufacturing systems. Journal of Manufacturing Systems, 47:93-106, 2018.

[91] P. Mahesh, A. Tiwari, C. Jin, P. R. Kumar, A. L. N. Reddy, S. T. S. Bukkapatanam, N. Gupta, and R. Karri. A survey of cybersecurity of digital manufacturing. Proceedings of the IEEE, 109(4):495-516, 2021.

[92] M. Pajic, J. Weimer, N. Bezzo, O. Sokolsky, G. J. Pappas, and I. Lee. Design and implementation of attack-resilient cyberphysical systems: With a focus on attack-resilient state estimators. IEEE Control Systems Magazine, 37(2):66-81, 2017.

[93] I. Linkov and A. Kott. Fundamental concepts of cyber resilience: Introduction and overview. In Cyber resilience of systems and networks, pages 1-25. Springer, 2019.

[94] Y. Chen, S. Kar, and J. M. F. Moura. Resilient distributed estimation: Sensor attacks. IEEE Transactions on Automatic Control, 64(9):37723779, 2019.

[95] F. Rosas, K. C. Chen, and D. Gündüz. Social learning for resilient data fusion against data falsification attacks. Computational social networks, 5(1):1-25, 2018.

[96] D. J. Miller, Z. Xiang, and G. Kesidis. Adversarial learning targeting deep neural network classification: A comprehensive review of defenses against attacks. Proceedings of the IEEE, 108(3):402-433, 2020 .

[97] A. Burg, A. Chattopadhyay, and K. Y. Lam. Wireless communication and security issues for cyber-physical systems and the internet-ofthings. Proceedings of the IEEE, 106(1):38-60, 2018.

[98] S. Karnouskos and F. Kerschbaum. Privacy and integrity considerations in hyperconnected autonomous vehicles. Proceedings of the IEEE, 106(1):160-170, 2018.

[99] B. X. Fang. Define cyberspace security. Chinese Journal of Network and Information Security, 4(1):1, 2018.

[100] K. Paridari, N. O'Mahony, A. El-Din Mady, R. Chabukswar, M. Boubekeur, and H. Sandberg. A framework for attack-resilient 
industrial control systems: Attack detection and controller reconfiguration. Proceedings of the IEEE, 106(1):113-128, 2018.

[101] C. Irvene, D. Formby, S. Litchfield, and R. Beyah. Honeybot: A honeypot for robotic systems. Proceedings of the IEEE, 106(1):61-70, 2018.

[102] C. Dong, Y. Xu, X. M. Liu, F. Zhang, G. R. He, and Y. Z. Chen. Hardware trojans in chips: A survey for detection and prevention. Sensors, 20(18):5165, 2020.

[103] D. Zhang, G. Feng, Y. Shi, and D. Srinivasan. Physical safety and cyber security analysis of multi-agent systems: A survey of recent advances. IEEE/CAA Journal of Automatica Sinica, 8(2):319-333, 2021.

\section{Biographies}

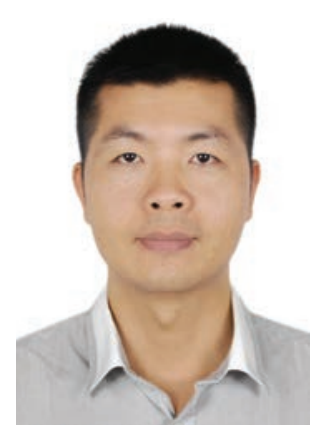

Zhenyi Chen received the B.S. and M.S. degrees in computer science from the College of Mathematics and Computer Science, Fuzhou University, China, in 2000 and 2005, respectively, and the Ph.D. degree in computer science from the Computer School, Wuhan University, China, in 2012. He is currently pursuing the second Ph.D. degree with the Department of Electrical Engineering, University of South Florida. His research interests include swarm intelligence, optimization and smart manufacturing. 


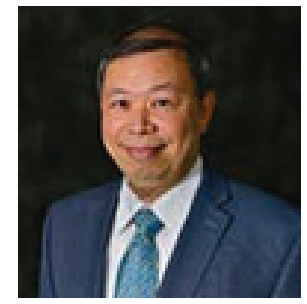

Kwang-Cheng Chen received the B.S. degree from the National Taiwan University, Taipei, Taiwan, in 1983 and the M.S. and Ph.D. degrees from the University of Maryland, College Park, MD, USA, in 1987 and 1989, respectively, all in electrical engineering. He was with SSE, Bethesda, MD, COMSAT, Clarksburg, MD, IBM Thomas J. Watson Research Center, IBM T.J. Watson Research Center, Yorktown and National Tsing Hua University, Hsinchu, Taiwan. From 1998 to 2016, he was a Distinguished Professor with the National Taiwan University, Taipei, Taiwan, ROC, where he was the Director of the Graduate Institute of Communication Engineering and the Communication Research Center and the Associate Dean for Academic Affairs of the College of Electrical Engineering and Computer Science from 2009 to 2015. Since 2016, he has been the Professor of electrical engineering with the University of South Florida, Tampa, FL, USA. He also actively participates in and has contributed essential technology to various IEEE 802, Bluetooth, LTE and LTE-A, 5G-NR, and ITU-T FG ML5G wireless standards. His recent research interests include wireless networks, multirobot systems, Internet of Things (IoT), and cyber-physical system (CPS), social networks and data analytics, and cybersecurity. Dr. Chen, an IEEE Fellow, received a number of IEEE awards, including the 2011 IEEE COMSOC WTC Recognition Award, the 2014 IEEE Jack Neubauer Memorial Award, and the 2014 IEEE COMSOC AP Outstanding Paper Award. He has actively participated in the organization of various IEEE conferences as the general/TPC chair/co-chair, and has served in editorship with several IEEE journals. 


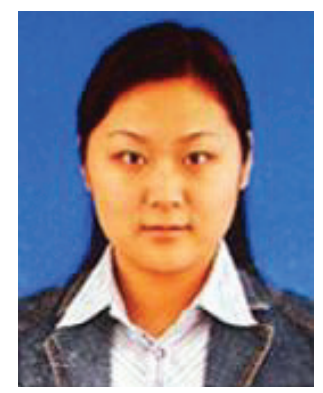

Chen Dong received her B.S. and M.S. degrees in computer science from Fuzhou University, China, in 2002 and 2005 respectively. She received her $\mathrm{Ph} . \mathrm{D}$. degree in computer science from the Computer School, Wuhan University, China, in 2011. She worked as a visiting researcher at the University of California, Los Angeles (UCLA), USA, from 2015 to 2016. She is currently working as an assistant professor at the College of Mathematics and Computer Science, Fuzhou University. Her research interests are artificial intelligence, big data and integrated circuit security and physical design.

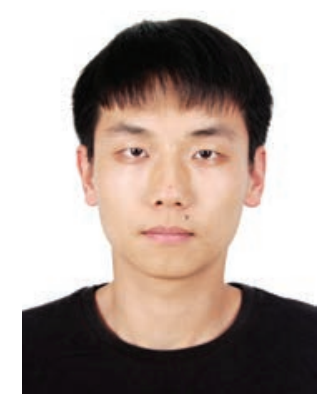

Zixiang Nie received B.E. degree at Beijing University of Technology, Beijing, China in 2016 and M.S degree at University of Florida in 2019. From 2019, he is a PhD student and research assistant at University of South Florida. His research involves computing solutions for multi-agent systems in context of smart factories. His research interests include wireless networked multi-agent systems, resilient multi-agent systems and cyber security. 